\title{
Signal Appropriation of Explicit HIV Status Disclosure Fields in Sex-Social Apps used by Gay and Bisexual Men
}

\author{
Mark Warner ${ }^{*}$, Juan F. Maestre ${ }^{\dagger}$, Jo Gibbs ${ }^{*}$, Chia-Fang Chung ${ }^{\dagger}$, Ann Blandford ${ }^{*}$ \\ "University College London, UK †Indiana University Bloomington, USA \\ mark.warner@ucl.ac.uk, jmaestre@indiana.edu, jo.gibbs@ucl.ac.uk, cfchung@iu.edu, a.blandford@ucl.ac.uk
}

\begin{abstract}
HIV status disclosure fields in online sex-social applications ("apps") are designed to help increase awareness, reduce stigma, and promote sexual health. Public disclosure could also help those diagnosed relate to others with similar statuses to feel less isolated. However, in our interview study $(n=28)$ with HIV positive and negative men who have sex with men (MSM), we found some users preferred to keep their status private, especially when disclosure could stigmatise and disadvantage them, or risk revealing their status to someone they knew offline in a different context. How do users manage these tensions between health, stigma, and privacy? We analysed our interview data using signalling theory as a conceptual framework and identify participants developing 'signal appropriation' strategies, helping them manage the disclosure of their HIV status. Additionally, we propose a set of design considerations that explore the use of signals in the design of sensitive disclosure fields.
\end{abstract}

\section{CCS CONCEPTS}

- Human computer interaction $(\mathrm{HCI}) \rightarrow$ Empirical studies in HCI; HCI theory, concepts and models.

\section{KEYWORDS}

signal appropriation, signalling theory, online dating, privacy unraveling, HIV disclosure, stigma, stigmatized populations

Permission to make digital or hard copies of all or part of this work for personal or classroom use is granted without fee provided that copies are not made or distributed for profit or commercial advantage and that copies bear this notice and the full citation on the first page. Copyrights for components of this work owned by others than ACM must be honored. Abstracting with credit is permitted. To copy otherwise, or republish, to post on servers or to redistribute to lists, requires prior specific permission and/or a fee. Request permissions from permissions@acm.org.

CHI 2019, May 4-9, 2019, Glasgow, Scotland Uk

(c) 2018 Association for Computing Machinery.

ACM ISBN 978-x-xxxx-xxxx-x/YY/MM...\$15.00

https://doi.org/10.1145/nnnnnnn.nnnnnnn
ACM Reference Format:

Mark Warner, Juan F. Maestre, Jo Gibbs, Chia-Fang Chung, Ann Blandford. 2018. Signal Appropriation of Explicit HIV Status Disclosure Fields in Sex-Social Apps used by Gay and Bisexual Men. In CHI Conference on Human Factors in Computing Systems Proceedings (CHI 2019), May 4-9, 2019, Glasgow, Scotland Uk. ACM, New York, NY, USA, 15 pages. https://doi.org/10.1145/nnnnnnn.nnnnnnn

\section{INTRODUCTION}

Research with people living with human immunodeficiency virus (HIV) (PLH) is still scarce within Human-Computer Interaction (HCI). Studies have focused on the design and development of mobile phone based interventions to improve antiretroviral medication adherence in patients (e.g., [45, 54, $65,69])$, and to promote HIV self-management (e.g., [15, 66]). Yet, these interventions do not directly address management of and coping with HIV-related stigma which can have a significant impact on the health of PLH [14, 78]. It is therefore critical that interventions designed to reduce stigma are studied. As stigma is a social construct, integrating interventions within existing online social environments may be a more effective strategy than developing stand-alone tools [82].

Recognising this, many sex-social apps used by MSM have integrated public HIV status disclosure fields in various different forms. Increased openness of HIV status information can help raise awareness, educate, and normalise HIV, as well as increasing people's known contact with PLH, all of which could help cultivate less stigmatised perceptions around the condition [14, 49, 82]. Increased disclosure can help reduce the spread of the virus, as it allows this at-risk population to make more informed sexual risk decisions. Yet, in online environments like these where people act to increase their own desirability $[24,31,86,89]$, some users may feel unable to publicly disclose due to the stigma that HIV attracts. This is especially pertinent amongst newly diagnosed users, half of whom report negative self-image and mental wellbeing concerns in the 12 months post-diagnosis [75].

The purpose of this study was to understand how current users are interacting with these HIV disclosure fields to help inform design approaches to reduce stigma, and promote openness around HIV disclosure, whilst also providing a 
supportive environment for those who feel unable to publicly disclose. We gain this understanding through interviews conducted with 14 PLH and 14 HIV negative MSM in London, UK.

These sex-social environments operate like markets [25, $39,49,82]$, with a supply and a demand and the need for users to 'signal' otherwise private information to each other to promote their own desirability. We therefore analyse this data using the conceptual framework of signalling theory. In doing so, we explore how our participants interact with these HIV signalling systems to manage the disclosure of their status, and make several contributions to the HCI literature:

- We provide a novel empirical contribution to HCI by applying signalling theory to sex-social applications. We use this theory to understand how MSM interact with HIV disclosure fields to manage their status using explicit and implicit forms of communication.

- We identify a form of technology appropriation which involves the cultivation of signals, helping users manage their privacy and fulfil certain psychological needs (e.g., belonging, relatedness).

- We propose a set of design considerations that draw on signalling theory to help reduce the stigmatising cost of HIV disclosure and help cultivate a supportive environment for potentially vulnerable users who may feel unable to disclose their status publicly.

\section{BACKGROUND AND RELATED WORK}

This section provides a background on HIV in the UK and explores the literature on HIV stigma, privacy, and disclosure. We then provide an overview of HIV disclosure fields in many of today's sex-social apps used by MSM. Finally, as we explore our interview data using signalling theory, we introduce this theory and discuss how it has been used within the field of HCI.

\subsection{HIV in the United Kingdom}

HIV disproportionately affects MSM in the UK, making up $54 \%$ of new diagnoses in 2016 [13]. Highly active antiretroviral therapy (HAART) means those diagnosed can often become 'undetectable', a term used to describe a PLH who has an undetectable level of the virus (viral load) in their blood. Once undetectable, extensive studies have shown the risk of HIV transmission is suppressed to effectively zero [67, 68]. To raise awareness of this fact, the term ' $U=U$ ' is commonly used, meaning undetectable equals untransmittable. Despite this, new infections are still occurring, in part due to those unknowingly infected with HIV $[9,36,63]$. In the UK, it is estimated that $13 \%$ of MSM living with HIV are unaware of their condition [13]. Alongside advances in treatments for those diagnosed, pre-exposure prophylaxis (PrEP) drugs to protect those who are at risk of HIV infection are becoming more widely available. These changes in the treatment landscape have created a multitude of different HIV statuses that individuals may identify as:

- HIV Negative. Those who have tested negative; however, those who are untested may also identify with this status.

- Negative on PrEP. Those taking this precautionary drug may identify as Negative on PrEP, and are likely to undergo regular HIV testing as part of the prescription process.

- HIV Positive. PLH who have a detectable viral load, and are still at risk of onward transmission during condomless sex.

- Undetectable. PLH who are on effective treatment who have an undetectable viral load.

- Not Known. Those who have not been recently tested, or have never been tested.

\subsection{HIV Related Stigma and Disclosure}

Stigma is a social construct based on the existence of "marks" or "traits" among individuals that make them be perceived as 'different' or 'not normal' from a socially-conceived perspective. According to Goffman [33], the bearers of such discrediting marks have 'spoiled social identities' that often lead to negative outcomes in social interactions such as rejection from others and self-isolation.

In the UK, around half of MSM who live with HIV reported feeling shame, guilt, and low self-esteem and/or self-blame in relation to their HIV status in the 12 months after diagnosis [75]. It is important to note, however, that HIV-related stigma exists as long as it is perceived by PLH themselves, and the degree to which stigma affects these individuals is related to the valence and salience of such perceptions [58]. Consequently, much of the research on stigma from the social sciences has been focused on the identification of coping strategies that allow individuals to ameliorate the negative effects of stigma in their lives. Such strategies include accepting the stigmatising trait and showing it to others (e.g., a gay man showing his sexual interest for other men in public), hiding the stigmatising condition (i.e., passing as someone who does not have the stigmatising trait), denying that the stigmatising condition applies to the individual, or denying that such stigma even exists [58].

Stigma has been linked to negative outcomes such as discrimination, prejudice, identity devaluation and deterioration of physical and psychological health $[10,40,51,52]$. In the case of HIV, stigma has been associated with depression and anxiety, especially when PLH have been the target of stigma in the form of discrimination or rejection [40, 59]. Stigma can also impact on access to and exchange of social support as PLH find it difficult to disclose their status to 
others due to fears of rejection $[44,62,83]$. Moreover, reduced HIV disclosure can risk increasing HIV transmission rates [64]. Yet research with certain ethnic groups in the US show PLH are often not disclosing [18] or are misreporting [17] their status to potential sexual partners met online, prior to meeting. While studies have shown that individuals are more comfortable discussing HIV status online, as opposed to offline [17, 70], the desire for privacy can create barriers to disclosure.

Previous research finds people develop different disclosure strategies for managing tensions between privacy to shield them from stigma, and disclose to facilitate the revealing of their status to gain support [73]. For instance, PLH have been found to develop signalling strategies in interpersonal communications to reveal their status. These signals "straddle the line" between being public and remaining private [19]. People may engage in verbal hinting, or use physical items as symbolic hints (e.g. HIV leaflets left on a coffee table) [71]. Research on revealing sensitive, potentially stigmatising information about the self in online social networks found similar strategies being adopted (e.g., sharing a blog post that someone else wrote on the sensitive topic) [3].

Researchers have tried to identify ways to minimise stigma at the individual (i.e., the bearer of the stigmatising trait) and at the population level (i.e., society). At the individual level, it has been found that PLH can alleviate the effects of HIV-related stigma by receiving peer support from "buddies" or "mentors" who are going through a similar experience $[10,79]$. At the population level, misinformation regarding HIV transmission and negative attitudes toward same sex orientation have been identified as the main factors that exacerbate HIV-related stigma [38, 40, 80]. Consequently, interventions have been designed to promote positive attitudes towards PLH. Brown et al. [14] completed a meta-analysis of 22 studies assessing interventions to reduce HIV-related stigma in the general public in the US and elsewhere. Results showed that providing information about HIV regarding transmission was not enough to minimise HIV-related stigma. Instead, there also had to be more direct contact and interaction with PLH.

\subsection{HIV Status Disclosure Fields}

There are a large number of sex-social apps designed for MSM, and the way in which HIV status information is managed differs between apps. However, broadly two approaches are used. The first, and most common approach is an explicit HIV disclosure design. Users are presented with a drop down menu containing a selection of HIV status options, and a last test date input field (see example: Figure 1 left). The HIV status options can vary between apps; for example, some apps provide users with a "Not Sure" option, whilst others do not. The second approach used primarily in the app Scruff

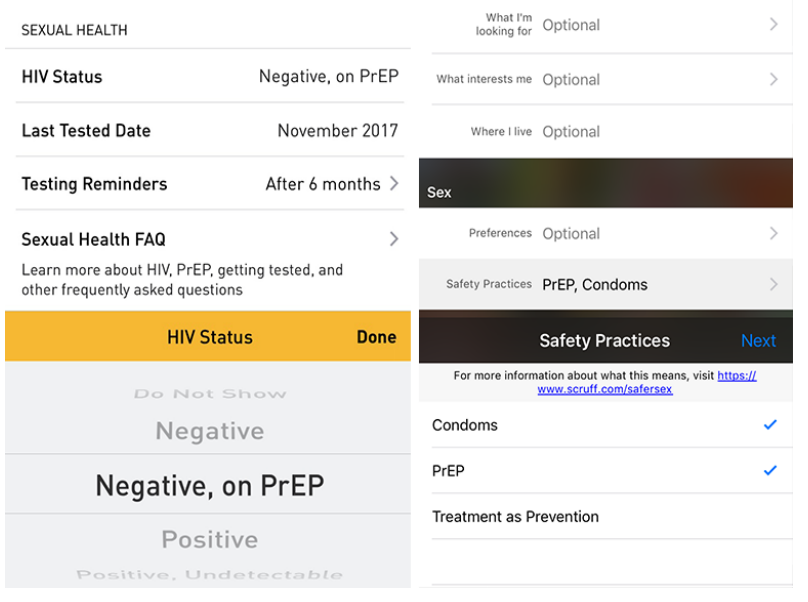

Figure 1: Cropped screenshots of the HIV disclosure fields in Grindr (left), and Scruff (right).

shown in Figure 1 (right) provides a safer sex practice disclosure field, allowing users to select from a range of safer sex practices (e.g., condoms, PrEP, Treatment as Prevention). This design allows users to select more than one option, and is ambiguous as to whether it is the safer sex practices of the individual disclosing, or what this individual is looking for in others, or both. Some applications also provide users with the option to identify with the HIV positive community. For example, Grindr provides its users with a tribe called "Poz", whilst Scruff allows users to state "I am Poz".

\subsection{Signalling Theory}

Signalling theory has its roots in economics, with signalling proposed as a mechanism for dealing with information asymmetry, where one transaction party holds more or better information than another. In Akerlof's [2] "Market for Lemons" work, he relates uncertainty and quality to provide a structured understanding of how dishonesty can negatively impact an entire market. To mitigate this, Spence [74] proposed signalling as a means for one party to reveal information to another to reduce information asymmetry. These are often referred to as 'signalling environments' [26].

In environments where individuals act to maximise their own value, Frank [29] described a phenomenon, the "full disclosure principle", which is the opposite of the "Market for Lemons" principle in that all individuals disclose to distinguish themselves from others, causing non-disclosure to become unsustainable [6].

Non-disclosure can then have what Peppet describes as a game-theoretic privacy unraveling effect [61] where undesirable assumptions develop around non-disclosures, effectively removing the element of control from privacy decisionmaking. This effect has been explored in an experiment involving information disclosure in a labour market [8], in a 
study of eBay Motor listings [50], and in a qualitative study into the disclosure of HIV status information in a sex-social application [82].

Whilst signalling in economics is focused on reducing information asymmetry between transaction parties, in biology the focus is on understanding how signals stay reliable. This is especially important as signalling is thought to be a key mechanism in biological evolution, from mate selection [87], to predatory avoidance [53]. If signals are so critical to evolution, how do they stay reliable? One way is for signals to be costly to produce, and therefore costly to fake. If a signaller uses excessive amounts of a finite resource, the "wastage" of this resource can act as a signal. Those with less of this resource would be unable to waste it, making the signal reliable. Zahavi coined this the "handicap principle" [87] referring to golfer's who "waste" their strokes to signal their ability.

Signals are also present in human communication [60], developing through a much faster process of cultural evolution [21]. Donath [21] identifies three types of signals that can occur in human social interactions. The first two are categorised as assessment signals, as the quality being signalled is inherent within the signal itself. These are (1) honest signals (e.g., handicap principle), and (2) index signals, which require the individual to possess the quality for the signal to be produced, e.g., golfers signal the strength in their arms by the quality of their swing. The final type is a (3) conventional signal which develops meaning through an established process of social convention. For example, the use of capitalised letters to signal shouting.

In this paper, we use Donath's [21] definitions of cues, signals, and evidence. She defines cues as anything used to infer some hidden quality or information. A cue becomes a signal where the sender has intention to convey the information, and evidence where the information is unintentionally conveyed. These communication mechanisms do not have to exist in isolation. For example, a person may signal some hidden quality, yet when the signal is received it may be evidence of a very different quality. As an example, a person may drive a large petrol sports car to signal their wealth, yet the signal receiver may use the car as evidence of the person's lack of concern for the environment.

\subsection{Signals and Cues in $\mathrm{HCl}$}

Researchers in HCI have drawn on signalling theory to understand and explain a range of online social technologies and behaviours. Its use often requires referencing to constructs from the market metaphor (e.g., costs). Although metaphors can help make sense of complex processes, they can impact on the shaping of people's social constructions of reality [7] which could have negative consequences in this context, such as the objectification of potential partners [39].
However, our work focuses on understand the management of HIV status information, rather than using signalling theory to understand relationship formations.

Although not explicitly applying signalling theory, previous HCI researchers have explored the use of digital artefacts to mediate signalling in various different forms. For example, a recent study found people repurposing emojis with different meanings to signal affection to specific groups or individuals [84]. Cultural evolution of emojis has also occurred; for example, the peach fruit emoji is now a common signal for buttocks rather than a type of fruit $[5,84]$.

In one study [48], online daters were provided with a limited number of virtual roses that they could send to other users to signal their interest. Limiting the number of roses per user created an artificial cost, increasing the reliability of the sender's signal.

Signals have also been explored in an online job market which identified "costly to fake" automatically generated content about a user as being more influential in impression formation than self-reported content [72]. However, selfreported data may not always be unreliable. The social aspect of online social networks can create a form of validation of claims that individuals make about their identity, with incorrect information being "called out" by other users in their network [42, 47, 81].

Online dating environments differ, consisting of pairwise interactions [56], limiting this type of social information validation. This can be particularly challenging when users misreport or embellish aspects of themselves to increase their online attractiveness [24]. In the absence of reliable information, people may develop other techniques to evaluate identity claims. One study found people using subtle "costly to fake" signals like poor spelling and grammar [24] to support claims related to education levels. Another found linguistic cues on dating profiles correlated with profile deceptions [76]. The unintentional nature of these signals mean they are more costly to produce, and could act as honest signals.

Signals can also be used to help foster changes in perceptions in social environments. Levy and Barocas' [49] research on designing against discrimination identified a gay sex-social app using 'pledges' to help cultivate stigma reducing norms around HIV. The app asked users to pledge to "Live Stigma-Free". Once made, the pledge appeared on the user's profile which also acted as a signal to other users, allowing them to learn more about one another.

Where information could be used to stigmatise and discriminate, one approach could be to suppress it. However, unintended consequences of this approach may disadvantage a wider set of users, as was observed around an initiative to remove questions related to job applicants' criminal conviction histories from job application forms. Initially it was 
thought this could help those with criminal records find employment, yet researchers found the absence of this information caused other cues (e.g., age, ethnicity, socioeconomic status) to be used by some employers as evidence of a candidate's likelihood of past criminal behaviour [20]. Another approach is to allow users individual choice to disclose, yet this too has limitations due to privacy unraveling effect that we discussed in section 2.4. This effect could cause those not disclosing to be assumed by others to be hiding something undesirable [61].

What all these signalling systems have in common is the need to be reliable. Systems that consistently support dishonest signalling lose reliability and break down. As Donath argues, signalling systems should develop to be beneficial for participants to produce reliable signals, yet costly to produce deceptive ones [22].

\section{METHODOLOGY}

Past research has identified the potential benefits of introducing HIV disclosure fields into sex-socials apps [14, 49, 82] whilst identifying potential privacy concerns with their "optional" disclosure design [82]. Signals can be cultivated in various different ways in social environments and so it is important to understand how people interact with these systems to identify potential improvements in their design. We conducted semi-structured interviews with both PLH and HIV negative MSM and in this section we present our methodology.

\subsection{Participant Recruitment}

This research was conducted as part of a wider study exploring online privacy, disclosure, and identity management behaviours around HIV status information with MSM living in the UK. Participants $(n=28)$ were invited to interview at our London campus. One participant who was unable to attend was interviewed via Skype. Our inclusion criteria for participants were (1) identify as being male, (2) aged over 18, (3) interested in having sex with men, and (4) active on at least one online social network or sex-social network. Only one participant reported no use of sex-social apps, but did report using at least one online social network. As this work was focused on sex-social app usage, this participant was excluded from our qualitative analysis.

Recruitment was performed primarily online, advertising on a number of online social and sex-social networks. We also advertised in local social meeting points (e.g., cafes). Participants were recompensed with a $£ 15$ voucher. Whilst online recruitment can create sampling bias towards people on those social networks, as our inclusion criteria included use of social/sex-social networks, we do not believe that this has an impact on our findings.

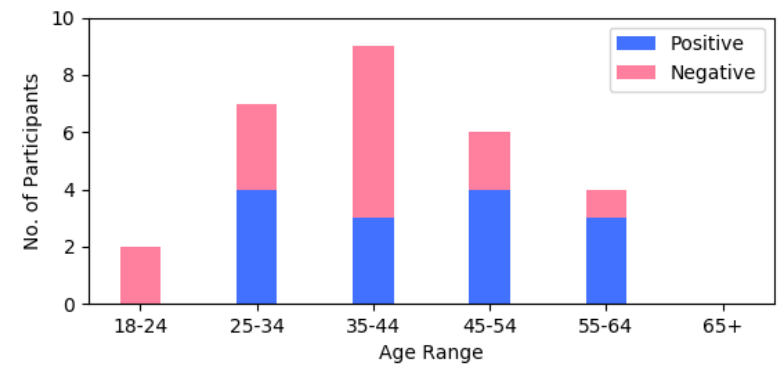

Figure 2: Bar chart showing age range of all participants, separated by HIV status $(n=28)$.

Our recruitment campaign ran for six months in 2017/18. A total of 44 men expressed an interest in the study. 28 were interviewed, eight arranged interviews but cancelled, and eight did not respond to follow-up e-mails. Of those interviewed, 14 reported to be HIV negative (five Negative on PrEP) and $14 \mathrm{HIV}$ positive (12 Undetectable). Figure 2 shows the age distribution of participants, showing a good distribution across ages except for under-representation in the age groups 18-24 and 65+. This can be partially accounted for by nearly $75 \%$ of gay and bisexual men newly diagnosed with HIV in the last 10 years being aged between 25 - 49 years [13], and Internet usage rates falling in people over the age of 60 [1].

We also asked each PLH how long they had been diagnosed to ensure we were capturing insights from PLH diagnosed for different periods of time. The PLH participants reported a broad distribution of years since diagnosis (less than one $(n=2)$, one to two $(n=3)$, two to five $(n=3)$, five to ten $(n=3)$, over ten $(n=3))$. Finally, we asked each participant the names of sex-social apps they used; $82.1 \%$ reported use of Grindr, $64.2 \%$ used Scruff, 28.5\% Tinder, 21.4\% Hornet, and $14.2 \%$ reporting using BareBackRT ('BBRT').

\subsection{Data Collection}

Interviews lasted between 41 and 88 mins $(M=63, S D=13.06)$ and were audio recorded, and transcribed by the first author. The interviewer made hand written notes during each interview, which were used to guide the analysis. The interviews were semi-structured and started with an "ice-breaker" question about participants general social and sex social network usage. This was followed by open questions relating to: online HIV disclosure behaviours, online social support for $\mathrm{HIV}$, online privacy, and online disclosure decision-making around HIV status. Participants were encouraged to talk freely about their experiences, perceptions, and behaviours. If they had a story, they were encouraged to tell that story if they felt comfortable doing so. Where a participant discussed something of interest with the interviewer, where 
Table 1: Abbreviated HIV status symbols

\begin{tabular}{cc}
\hline Reported HIV Status & Abbreviated Symbol \\
\hline Negative & Neg \\
Negative on PrEP & PrEP \\
Positive & Pos \\
Undetectable & $\mathrm{U}=\mathrm{U}$ \\
\hline
\end{tabular}

appropriate they were asked to elaborate (e.g. "could you elaborate a little on what you mean by that?", "could you explain that?").

\subsection{Data Analysis}

An initial inductive thematic analysis was conducted by the first author [12], and was interleaved with data collection. This allowed him to immerse himself in the data and reflect on the interviews. Subsequent interviews were adapted as knowledge and understanding of the topic developed. Open coding was performed across each transcript using mindmaps to visualise codes across participants in support of axial coding, and to explore differences in disclosure behaviours and tensions. Use of visualisation tools at this stage of the analysis is suggested by Braun and Clarke [12], and mindmaps have been previous used in HCI research (e.g., [4]).

During this analysis, we identified subtle forms of privacy and disclosure management behaviours, as well as assumptions developing around non-disclosures which we suspected were the effects of the privacy unraveling [61]. As Peppet's unraveling effect has its roots in signalling theory, we reviewed this theory and used it to develop a thematic theoretical framework to perform a deductive analysis. We developed codes based on the following themes: signalling costs; signals that develop around non-disclosures; reliability of HIV status disclosures; and signalling strategies used to manage HIV disclosure. Donath's cues, signal, and evidence constructs were separately coded [21]. The first author then performed a second round of coding as part of the deductive analysis using the developed thematic framework.

Each participant number is followed by an abbreviation of the participant's self-reported HIV status (see: Table 1). In abbreviating PLH we used 'Pos' to refer to those who did not report being undetectable, and ' $\mathrm{U}=\mathrm{U}$ ' to refer to those who did report being undetectable. When discussing participants more generally, we refer to them as being either PLH (i.e., HIV positive/undetectable) or negative to reduce complexity.

\subsection{Ethical Considerations}

Due to the sensitive nature of HIV, we designed our study to allow each participant to reflect on the details of the study prior to making contact with the research team. To achieve this, we published a website which contained a detailed

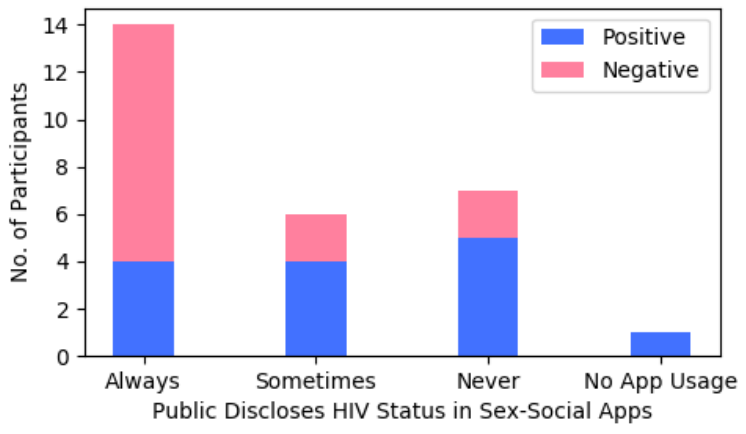

Figure 3: Bar charts showing participants' $(n=28)$ reported public HIV disclosures in sex-social apps.

overview of what taking part would entail, and linked all our advertising campaigns to this website. Before each interview, participants were handed an information sheet detailing the study, and were then asked to complete an informed consent form. Interviews were anonymised during transcription by removing any names, unique references, or non-relevant detail that could be used to identify the participant. This study protocol was submitted and approved by the University College London ethics board, reference number: 11699/001.

\section{FINDINGS}

This section presents the findings from our inductive and deductive analysis. We first present the findings from our initial inductive analysis in section 4.1, where we explore our participants disclosure decision-making behaviours, and tensions that were identified. We then present the findings from our deductive analysis (section 4.2 onward) performed using the conceptual framework of signalling theory.

\subsection{HIV Disclosure Behaviours and Tensions}

Disclosure of HIV status information within sex-social apps was much less common amongst PLH (see: Figure 3). Of the subset who reported never publicly disclosing, most disclosed when interacting in private chats, although one PLH reported never disclosing his status. When discussing HIV disclosure, fear and stigma were often at the centre of participants' decision-making, with tensions between people's values, needs, and goals affecting disclosure. In this section, we first discuss the direct effect stigma has on disclosure, and then explore some of these tensions.

4.1.1 Fear and stigma. A prominent reason for keeping HIV status undisclosed was stigma. Primarily, this took the form of verbal abuse or loss of sexual opportunity. Whilst not all PLH reported this, the impact it had on those that did often caused them to remove their status from public display. HIV related stigma can be particularly challenging for those 
who already feel stigmatised (e.g., due to age, sexuality). For instance, $\mathrm{P} 21^{\mathrm{U}=\mathrm{U}}$ describes his HIV status as an additional "variable", on top of his already stigmatised older age: "so the variables for me tend to be about age and so this introduced a further variable".

For many, stigma and fear of rejection caused them to keep their HIV positive status undisclosed; for others it was a way of avoiding HIV related rejection. $\mathrm{P} 1^{\text {Pos }}$ for example was active on the dating app Tinder. Whilst Tinder does not provide an explicit field for reporting HIV status, he disclosed in the free text field on his profile. This created an organic filter, causing those who may reject him because of his status to avoid making contact: "it kinda cuts out the bullshit as well you know, [..] if you've got an issue with it, then, then don't go any further".

4.1.2 Threat to identity. Those able to disclose publicly had often been living with HIV for some time, and had accepted it as part of their identity. Yet, in the period after diagnosis, life can be turbulent. Newly diagnosed PLH reported feeling isolated and fearful of how people's perceptions of them would change. Some used sex-social apps anonymously to help them find others for support. For example, $\mathrm{P} 24^{\mathrm{U}=\mathrm{U}}$ said that: "[I] setup a profile initially that was saying [.. who else is +?", whilst for $\mathrm{P} 19^{\mathrm{U}=\mathrm{U}}$, these environments provided a means for him to test the social response of his status in a less accountable environment, saying: "I remember thinking at the time, this is a way for me to, in essence to test the waters, [.. to test what the reaction was".

HIV disclosure was often not consistent across all life contexts, leading to concerns that disclosure in one context could risk "outing" them in another. While HIV was part of $\mathrm{P} 27^{\mathrm{U}}=\mathrm{U}$ 's sexual identity, he did not want it forming part of his identity at work and was concerned his work colleagues would discover his status through sex-social apps: "where I work there is a lot like, groups of gay people, there's a lot of groups like Pride and all sorts of kind of stuff and it's like, it starts to really blend the lines between dating and work" $\left(\mathrm{P} 27^{\mathrm{U}=\mathrm{U}}\right)$.

4.1.3 Value conflicts. Some participants discussed their values of openness and honesty as reasons for publicly disclosing their status. This was reported by both PLH and negative participants: "I was just uploading the new profile and it just seemed, it was a step that was there that just seemed appropriate and you know it was aligned with my concepts of transparency." (P12 $\left.{ }^{\mathrm{PrEP}}\right)$. For PLH, being open about their status would often come at a much higher cost. Abuse and loss of sexual opportunity caused some to view their status as "spoiling" their identity [33]. Keeping their status publicly undisclosed allowed for greater control over access to this aspect of their identity and how it was relayed to others. Participants would often prefer disclosing in private chats as this allowed for a relationship to develop prior to disclosure. This was described by $\mathrm{P} 9 \mathrm{U}=\mathrm{U}$ who said: "I think it [Undetectable status] probably does scare people away a little bit even if they are relatively clued up, but then I kind of think it's less when you've been talking for a bit, [..] it's not necessarily the first thing you want others to see".

Although a more selective disclosure approach provides greater control, participants were often conflicted between maintaining privacy around their status, and being more open to help normalise HIV. Public disclosure was seen as a way to normalise HIV, and to make it more accepted and less stigmatised. For example, $\mathrm{P} 23^{\mathrm{U}}=\mathrm{U}$ who was very open about his status both offline and online stated: "it's about normalising things, it's about, when something becomes usual people stop having an issue with it, people stop noticing it". Those keeping their status private would often report feeling guilty at not taking a more open approach, perceiving their silence to be contributing to the stigma around HIV.

Like those motivated to disclose to normalise HIV, others were driven by their values to educate. There is still a considerable amount of out-of-date discourse around HIV, and a number of our PLH participants reported spending time curating their profiles with educational HIV information, as well as acting as an educator in private chats. Yet, some became frustrated with having this role as it reduced sexual excitement and detracted from their primary goal of dating and finding prospective sexual partners. $\mathrm{P} 24^{\mathrm{U}=\mathrm{U}}$ who originally disclosed publicly became frustrated at having conversation about his status, stating: "I just thought no, I can't be bothered with all the, having to explain [..] so I just took it off, so I choose to disclose now when I talk to somebody".

\subsection{Changing Cost Dynamics}

As discussed previously, the stigma that HIV attracts means that for many MSM living with HIV, disclosure of their status comes with a high social cost. This is especially significant in sex-social environments, as HIV historically led to fear, anxiety, and stigma around sex for MSM, and is often still perceived that way today.

Whilst being HIV negative remains low cost, the time elapsed since being tested often had a negative effect on disclosure cost. As P10 ${ }^{\mathrm{PrEP}}$ described: "I'm always a bit dubious when last time tested is like 2016, that's always a bit "ok, you seem to think that it's ok [to test] every year"". An out-of-date test could be used as evidence that the signaller was not taking care of their sexual health. Some participants recognised this and instead removed the last test date from their profile to avoid this acting as an undesirable cue.

The increasing use of PrEP within this community has the potential to increase the cost of a negative status for those not on PrEP. The date of last test is perhaps less significant when the profile is disclosing PrEP use, as they are 
actively protecting themselves from HIV between tests even when engaging in condomless sex. Moreover, as part of the PrEP prescription, they are likely to be undergoing regular testing. For example, $\mathrm{P} 8^{\mathrm{Neg}}$ stated: "PrEP has kind of reduced my status, so before PrEP being negative was as good as you could get, but now with negative on PrEP that's like an $A^{*}$ so I've been demoted, irritating". However, not all participants perceived PrEP status as being a low cost signal. Some felt unintentional signals had developed around its use, evidence of an increased willing to engage in condomless sex, a behaviour known as "barebacking". $\mathrm{P} 23^{\mathrm{U}}=\mathrm{U}$ reported the reason his husband did not disclose being on PrEP: "He said there's a perception, and he's right, in the gay community that if you're on PrEP, then you are therefore automatically into bareback sex and he's not, so he doesn't want the solicitations from people who are going to assume that".

Along with increasing the cost of a negative status, increased usage of PrEP within the community, combined with the advent of $U=U$, appears to have reduced the cost associated with an HIV positive disclosure. Our findings suggest this has resulted for two reasons: Firstly, PrEP taken correctly prevents the acquisition of HIV which allows people to be in control of their own HIV risk, reducing fear. Secondly, in the process of learning about and being prescribed PrEP, many participants reported becoming much more aware and knowledgeable of wider HIV related issues. One participant even suggested that it had resulted in a cultural shift: "I think because the culture has changed so much because of PrEP and the recent findings that if you're undetectable then you just can't pass it on that guys are a lot more relaxed about it" $\left(\mathrm{P} 25^{\mathrm{U}=\mathrm{U}}\right)$.

\subsection{Non-Disclosure Cues}

In this section, we look to understand the social impact nondisclosures have on both PLH and negative participants, and whether the privacy unraveling effect causes non-disclosures to act as cues. Our analysis identified a number of instances where PLH were concerned that by leaving their HIV status undisclosed, this would act as a cue which could be used to infer their HIV status. As an example, $\mathrm{P} 9 \mathrm{U}=\mathrm{U}$ removed his status after he noticed a drop in responses: "when I took it down I thought everyone's going to think I'm positive. Maybe that's one of those things where like, seeing it as a whole is different from what it personally means to you". Whilst this fear did not result in $\mathrm{P} 9 \mathrm{U}=\mathrm{U}$ disclosing, $\mathrm{P}^{\mathrm{Neg}}$ did choose to disclose, in part, because of a fear of being perceived as being HIV positive: "I put myself as negative also because I think there's a growing stigma, it might be just in my head, but if you don't put anything, it seems like you're trying to hide something, maybe, but that may just be in my head but I thought, I am negative so I might as well say so".
Whilst some participants felt their own non-disclosure could act as evidence of an undesirable status, they did not always perceive this when viewing other profiles with undisclosed HIV statuses. Above we showed how $\mathrm{P}^{\mathrm{Neg}}$ disclosed his status through fear of being stigmatised, but when asked how he perceived this in others he stated: "I think if you don't have anything, my initial reaction is, they just haven't put it in, [..] I don't think "oh they're positive and they don't want to say anything".". Some participants also felt non-disclosure assumptions were reduced where other profile fields (e.g., age, height) were incomplete: "you get those people who don't put anything on their profile, have nothing or just one line, so they don't file their age their height anything like that, umm but I think for people that bother to fill in, you know a reasonable section of the profile information, they answer their HIV question" (P8 $\left.{ }^{\mathrm{Neg}}\right)$.

\subsection{Signal Reliability}

When users publicly signal their HIV status information in sex-social apps, there is typically no form of verification that the status disclosed is accurate. In this section we explore the reliability of these signals by looking at what makes a signal reliable, and how our participants act to increase their trust in the signals of others.

The most common statuses being signalled amongst participants were either HIV negative or HIV negative on PrEP. It was not uncommon for the reliability of these signals to be questioned due to the trust that is required in the signal sender: "in a way [public disclosure] is bad because you're relying on that person to be honest" ( $\left.\mathrm{P} 2^{\mathrm{Neg}}\right)$. The reliability of HIV negative signals was often questioned, especially when the time since last test had elapsed passed a certain point. The change in cost dynamics discussed in section 4.2 appeared to affect the reliability of HIV status information: "I kind of think, if [..] it's [last HIV test] a year or two ago well, it doesn't mean anything really, you don't know is what you're saying" (P8 $\left.{ }^{\mathrm{Neg}}\right)$.

The relatively low-cost associated with a negative on PrEP status means individuals who possess a high cost status may become incentivised to misreport their status to avoid being stigmatised. Whilst non-disclosure is an option, the privacy unraveling effect discussed in section 4.3 can also result in non-disclosures becoming stigmatised. We found a number of PLH had misreported or seen others misreporting their HIV status. For example, one PLH who used Scruff stated: "I've unticked the treatment one [Treatment as Prevention], because that's implying that you're on treatment [HIV positive], so I didn't want to just broadcast that [..] I left PrEP on there because I' $m$ in that same category" $\left(\mathrm{P} 27^{\mathrm{U}=\mathrm{U}}\right)$. However, for a number of other participants the disclosure options provided by Scruff created uncertainty causing them to either abandon the feature, or tick all the options available: "it isn't 
very clear whether it's what you engage in, or what you expect your partner to engage in, so I tick them all because I think if somebody who is on PrEP wants to approach me, then yes there would be a conversation" $\left(\mathrm{P} 28^{\mathrm{U}=\mathrm{U}}\right)$. A number of other participants reported that they felt being negative on PrEP was very similar to being undetectable. Both are unable to pass on the virus, and both are taking very similar medication. This caused some of our undetectable participants to misreport or contemplate misreporting their status as negative on PrEP.

Typically, the most reliable signal that was reported was an HIV positive or undetectable status. As these signals come with high social costs, the perception was that a person would be unlikely to signal these statuses unless they were accurate. For men looking to engage in unprotected "bareback" sex, the reliability of these signals was much more important than to those with a preference for condoms. For these individuals, signals with higher reliability were much more attractive: "I think the one thing about the majority of the guys in my fraternity being HIV positive, we're getting regularly checked for other diseases, which in itself umm, negative guys have said "well, you can tell you're quite attractive to a negative guy because you're a pretty safe bet" do you know what I mean?" (P16 $\left.{ }^{\mathrm{U}=\mathrm{U}}\right)$.

Participants felt inhibited in their ability to build trust online as opposed to offline. Consequently, participants found it more difficult to evaluate the reliability of information more generally, and in particular the reliability of HIV status information: "anyone online can say "yeah I'm on PrEP, I'm positive that I've got an undetectable viral load" but you don't know, but I think if you're meeting with someone and you're going out with them and you're dating them, you build that trust" (P4 $\left.{ }^{\mathrm{Neg}}\right)$. Some participants reported evaluating other aspects of a user's profile, attempting to establish the reliability more generally: "there's that element of trust that actually that picture of the 29 year old is actually, one, that they are actually 29, rather than 45, positive, obese rather than, do you know what I mean? umm, which at some point you're gonna [sic] find out and in a way, if [..] that and that don't match up to reality then you might kind of think, well hang on a minute, does this [HIV status]?" (P2 $\left.{ }^{\mathrm{Neg}}\right)$.

\subsection{Signalling Strategies}

4.5.1 Costly to produce. One strategy used by participants was the "costly to produce" strategy. As discussed in section 4.4, HIV statuses with high social cost are often deemed more reliable. Although being undetectable was not seen as the most costly, some felt the cost was high enough to be costly to produce: "I just don't understand why you would lie to say you're undetectable when you could just lie to say you're negative, do you know what I mean?" (P3 $\left.{ }^{\mathrm{PrEP}}\right)$. Yet, others felt a positive status was "the only really believable claim", suggesting "everything else should be taken with a pinch of salt" (P8 $\left.{ }^{\mathrm{Neg}}\right)$.

This "costly to produce" signal strategy was particularly effective with HIV negative participants who engaged in regular unprotected "bareback" sex, as signal reliability for this group was much more important than for those using condoms. Yet, regular condom users also saw the benefit of an undetectable status and its reliability: "I would feel safer sleeping with somebody that was HIV positive and told they were undetectable and I was on PrEP then I would if I wasn't and somebody was just randomly saying that they'd had a [HIV] test" $\left(\mathrm{P} 10^{\mathrm{Neg}}\right) . \mathrm{P} 23^{\mathrm{U}=\mathrm{U}}$ recognised the benefit his public status could afford others, and was very explicit in relaying this information: "One of the things that I state on my profile [..] is: "do you know what? you're so much safer having sex with somebody who knows themselves to be positive and undetectable than having sex with somebody that tells you that they are negative"".

4.5.2 Countersignaling. As we discussed in section 4.3, whilst many of the sex-social apps provide a non-disclosure HIV status option, where users choose to keep their status undisclosed, stigmatising cues can develop. A number of PLH who chose not to publicly disclose their status performed "countersignaling". They acted to reduce the strength of these non-disclosure cues by limiting disclosure across other parts of their profile (e.g., weight, interests). This was explicitly described by $\mathrm{P} 26^{\mathrm{U}=\mathrm{U}}$ who stated: "mine's very limited in what I fill out anyway so I think it just says my height and my position and that's it [..] so I guess if you answered all the other questions and excluded that one [HIV status] then maybe someone might ask questions", when asked whether he restricted disclosure of these other fields on purpose, he replied: "yep, (laughter) [..] it's that thing of I just don't want everyone kind of knowing about everything I guess"

Whilst $\mathrm{P} 26^{\mathrm{U}=\mathrm{U}}$ was explicit in his intention to countersignal the effects of unraveling, other participants who preferred to keep their HIV status undisclosed also described limiting disclosure across their profile: "my profile is exceptionally thin on the ground, it's basically got a picture of me where you can't see all of my face, you can see my mouth, it's just got my age, my background, my ethnicity, my height and that's all" $\left(\mathrm{P} 24^{\mathrm{U}=\mathrm{U}}\right)$.

PLH who preferred to disclose their HIV status in more intimate one-to-one interactions often reported developing educational strategies to counter the stigmatising effects of their HIV positive status. Participants reported various means of achieving this. $\mathrm{P} 21^{\mathrm{U}=\mathrm{U}}$ used a feature in Grindr called 'saved messages' to share HIV related information, and a link to an online video ${ }^{1}$ related to undetectable: "the first one is quite a short paragraph which is [..] do you know

\footnotetext{
${ }^{1}$ E.g., https://youtu.be/-Vew9W_dbkg
} 
what undetectable means? Do you understand what that's all about?, the second is more kind of detailed, a statistical kind of thing, and the third one is a link to a YouTube video". $\mathrm{P} 27^{\mathrm{U}=\mathrm{U}}$ used an image containing information related to evidence based research on undetectable transmission rates: "I have a photo that I send them which is from a recent study which is basically a screenshot of text".

4.5.3 Unraveling as an intentional signal. So far, we have discussed the effects of privacy unraveling in terms of its potential to disadvantage users and limit their disclosure choice. However, one participant reported exploiting the effect to his advantage. $\mathrm{P} 27^{\mathrm{U}=\mathrm{U}}$ wanted to disclose his status to others on Grindr to find other PLH that he could relate to. This was challenging due to the location-aware nature of the app creating a context overlap, meaning information disclosed in his online sex-social life could leak across into his professional life. In contrast to participants who engaged in countersignaling to reduce the effect of unraveling, $\mathrm{P} 27^{\mathrm{U}=\mathrm{U}}$ exploited this effect, recognising that by leaving his HIV status undisclosed it would send a subtle signal of his HIV positive status to other PLH, whilst limiting information leakage to his work colleagues: "I don't want to lie, and I also need to find a way to let other people know [of his HIV positive status] that are in the same scenario [..] it's like finding common ground, but you can't find common ground if you're not going to share anything and then you don't want some idiot at work, you know flying off the handle kind of thing, it's a bit of a fine line [..] I think Grindr has got it the best because you can just put nothing, and everyone puts something, so it's very subtle but you know, it's actually not really subtle" $\left(\mathrm{P} 27^{\mathrm{U}=\mathrm{U}}\right)$. Here we can see the unraveling effect being exploited as an intentional signal, allowing him to regain some control over the disclosure of his status. This strategy afforded him a new type of plausibly deniable HIV disclosure, allowing him to manage the ill effects of information leakage across contexts.

4.5.4 Status Repurposing. The final strategy that we identified was the repurposing of signals. Whilst the HIV status disclosure options that many sex-social apps provide seem very explicit and rigid in their meaning, we identified some users attempting to change their meaning. For example, a number of our participants reported undetectable users disclosing as negative on PrEP to reduce disclosure cost and to signal a desire to engage in bareback sex. A number of our participants perceived PrEP users as being more promiscuous and into higher risk sex than non PrEP users. This repurposing of negative on PrEP status was explicitly described by $\mathrm{P} 20^{\mathrm{U}=\mathrm{U}}$ : "I actually put negative on PrEP on my profile for a bit, [..] I think some positive people might use that as code [..] it's not used by positive people as a way of slipping under the radar of bigots umm, it's definitely used by people who want bareback sex, to advertise that, I think that's something that people do". Supporting this, a number of undetectable participants stated strong similarities between being undetectable, and negative on PrEP: "I like kind of see them as, I kind of think that if you're undetectable it's kind of the same as being on PrEP, like you're protecting other people and yourself" $\left(\mathrm{P} 26^{\mathrm{U}=\mathrm{U}}\right) . \mathrm{P} 16^{\mathrm{U}=\mathrm{U}}$ reported seeing a number of undetectable users disclosing as negative on PrEP: "I just sort of felt that, is it HIV guys actually saying they are negative to make themselves more attractive? They're on PrEP so I'm looking after myself and all this stuff, and therefore can use that as a lever, I might be wrong".

\section{DISCUSSION}

In this paper we use the theoretical framework of signalling theory to understand how MSM manage HIV status information using the HIV status disclosure options in many of today's sex-social apps. We use these findings to discuss ways in which design can be used to support the reduction of stigma to lower the cost of disclosing for PLH. Yet, we recognise this is a long-term goal. As such, we explore the role of design in making these environments more supportive for those who feel unable to publicly disclose their status due to the stigma attached to HIV, as well as those wanting to be open about their status. These design considerations are developed using the signalling strategies identified in our findings.

\subsection{Cultivating Stigma Reduction}

Online sex-social apps are often well subscribed, with large numbers of prospective partners available. Additionally, the effort required to reject users is low, often performed with a single finger gesture. As such, the social and physical costs needed to reject users are minimal. This can make public disclosure a difficult proposition for PLH. As our findings show, the social cost of an HIV positive status is high due to its perception as a socially undesirable attribute. Public disclosure can become unaligned with people's initial interaction goals of appearing desirable to others [32, 82]. Our findings support this, with a number of PLH preferring to disclose in more intimate one-to-one interactions after rapport had developed, which can increase the social cost of rejection. As discussed in section 2.2 there are many health and social benefits to public HIV disclosure, and sex-social apps are well positioned to help reduce these costs through design. Previous attempts have been made to design out stigma with community 'pledges' [49]. These could benefit online environments like these in two ways: firstly by placing HIV stigma into the social consciousness of users, and secondly the 'pledge' could act as a conventional signal to others, which may help PLH feel more confident in disclosing to users signalling this 'pledge'. 
1. Cultivating perception change around HIV should be a long-term goal within these environments. Designers could consider developing stigma reducing signalling systems, such as "living stigma-free" pledges.

Education as a means of updating out-of-date views around HIV is used to help reduce stigma [46, 85], lowering the cost of disclosing an HIV positive status. Some participants took on the role of educator, both in public disclosures (e.g., adding educational information on a profile), and in one-to-one interactions. Yet, we found frustrations developing with having this role, which lead to users developing strategies to speed up and regulate this process using existing app features. While some of the sex-social apps integrate educational information, it is often outside the daily user interactions, and is not easily accessible for sharing with others.

2. Designers could explore embedding educational HIV information and tools within interactions to help marginalised users in educating others. Those less aware of HIV related terms could become aware of them through their everyday interactions in the app.

3. Reminders of the benefits of public HIV disclosure could help both PLH and negative users. For PLH, it could provide disclosure purpose (e.g., stigma reduction). Yet, care is needed to avoid placing pressure on those who feel unable to disclose. Guidance could reassure recently diagnosed PLH that public disclosure is not expected. For HIV negative users, this information could help them recognise the public 'good' that PLH who disclose publicly are having within their community.

\subsection{Signal Appropriation}

Researchers have studied how users self-present in various online spaces, from social networking sites [41, 57, 77, 88] to online dating environments [24, 31, 86, 89]. They have also looked at how online users perceive the impressions of others [28]. However, it is only recently that researchers have started looking at how users navigate self-presentation in online environments that are not designed to support their needs, especially amongst stigmatised populations [3]. Explicit disclosure fields provide support for users motivated to disclose their status publicly, but for those that are not, these fields can limit their ability to keep this information private. As a consequence, we find users appropriating these fields using 'signal appropriation' strategies. We use this term to describe the use of signals as a mechanism for appropriating social technologies.

The explicit HIV disclosure design shown in Figure 1 (left) provides users with a non-disclosure option, yet we find evidence to support previous research [82] that these fields do not provide sufficient disclosure choice. The low disclosure cost, and subsequent ease in which a HIV negative status is disclosed, can cause privacy to unravel around these fields when undisclosed, becoming evidence of a signaller's undesirable HIV status. The ambiguity of the second design approach shown in Figure 1 (right) appears to limit this unraveling effect. The lack of definition can support users in developing their own interpretations and meanings around designs [11, 30,37], and support users in less direct forms of disclosure to reveal stigmatised aspects of their identity [3, 19, 23, 71]. Yet, if ambiguity is implemented through increasing complexity (e.g., providing many possible variations of disclosure), this can confuse users, as it becomes harder for signals and their meaning to cultivate and gain consensus within a given social environment.

In part, the lack of complexity around privacy unraveling contributes to its privacy invasive nature, yet this same simplicity allowed for this effect to be appropriated to reveal its affordance properties. The effect can provide PLH with a means of connecting with one another for support and feelings of relatedness and belonging - shown to help reduce stigma [10,79] - without having to explicitly disclose their status. This is especially pertinent for users who fear the social risk of post-disclosure rejection [62, 83]. Haimson et al. [35] found anonymity being used to create a less socially risky environment for information seeking and support, a finding reflected in our work. But subtle signals can provide an alternative approach to disclosing sensitive information online within existing, identifiable social networks. This behaviour is not isolated to the disclosure of HIV status. Andalibi et al.'s [3] research on communicating pregnancy loss found women using digital artefacts as cues to signal their loss to others. This too afforded them plausible deniability which allowing users to be selective in who they would respond to if asked directly about their loss.

This has another potential benefit. The location-aware nature of sex-social apps can lead to context collapse [55]. For instance, work colleagues may view each others' profiles while co-located at work. Research found PLH protect their workplace roles by having a preference for privacy [27]. The affordance properties of privacy unraveling could help protect this form of context overlap, while allowing PLH to signal their status. Many of the current explicit disclosure fields provide binary disclosure states (Disclosed/Undisclosed). If subtle signalling systems were developed around HIV disclosures, this transition from one state to another could be made more fluid, providing a continuum of disclosure regulation through indirect, ambiguous disclosure behaviours, consistent with previously identified offline disclosure strategies $[19,71]$. 
4. Designers should consider the privacy implications of binary style explicit disclosure fields. Non-complex ambiguity could be used as a resource to limit the effect of unraveling, and promote a socially cultivated signalling system. These could be used to develop subtle signals to support marginalised users in making less direct disclosures. This could help them connect with similarly positioned users for support and to fulfil psychological needs of relatedness and belonging, whilst respecting privacy.

\subsection{Harmful Signal Cultivation}

We also identified various other signal appropriation behaviours occurring within these HIV signalling systems. Like the repurposing of emojis to signal affection [84], some participants repurposed both low-cost HIV statuses (e.g., "Negative, on PrEP") and high high-cost HIV statuses (e.g., "Undetectable status") to signal sexual risk preferences (e.g., "bareback sex"). Where these signalling systems, which rely primarily on conventional signals, were unaligned with the user's needs, we found attempts to cultivate new meaning around HIV status cues by changing social convention around them. Yet, this form of signal appropriation can have a detrimental effect on honest signallers. For example, those signalling PrEP use may be harmed by their status being interpreted as evidence of wanting "bareback sex", leading to increased stigmatisation around its use [34, 43]. The effects of this could be MSM at risk of HIV not seeking PrEP as a result of this stigma [16]. Where dishonest signals develop, Donath [21] suggests finding ways of making these signals more costly to produce. However, this can be challenging with conventional signals, and while some research has explored designing in artificial costs $[48,82]$ this may not always be possible. An alternative approach would be to design in countersignals to support users to reduce these stigmatising signals, and help prevent "devolution" of these conventional signals. The second design shown in Figure 1 (right) allows for this type of countersignalling, providing users with the ability to disclose a preference for both PrEP and condoms.

Countersignals were also developed naturally by PLH, helping them counter the undesirable effects of privacy unraveling. Some PLH who preferred to keep their HIV status undisclosed would limit the disclosure of other information on their profile to reduce the strength of the privacy unraveling signals. However, this meant limiting the non-HIV related information disclosed which could disadvantage them. Although past research has found disclosure of fixed fields in dating applications to be unrelated to perceived attractiveness [28], many modern sex-social application allow users to filter on these fixed fields (e.g., age, height). If these fields are left undisclosed, the user may experience reduced visibility to others.
5. Designs could monitor these environments to identify the repurposing of HIV status options, designing in countersignals to mitigate stigmatising signal appropriations (e.g., allowing PrEP users to signal a preference for condoms).

\section{LIMITATIONS}

Our focus was on understanding online privacy and disclosure behaviours around HIV status in the MSM community in the UK, yet most of our participants were recruited from London which has a higher proportion of MSM, and higher rates of HIV. Therefore, our participants may not be representative of the wider MSM UK population. We did not control for ethnicity, and whilst this was only raised once in the study, different signalling behaviours may cultivate differently within different ethnic groups, and in different cultural settings. Therefore, it is important to recognise that while the signal appropriation principle presented in this paper is likely to generalise, the specific signalling system cultivated may not.

\section{CONCLUSION}

HIV disclosure fields in sex-social apps used by MSM are now commonplace, yet tension exists between the benefits they can provide (e.g., reduce stigma through formalising of language [49], increasing direct contact and interactions with PLH [14]), and the privacy concerns of those marginalised users who feel unable to publicly disclose their status in such environments. Designers therefore have the challenging task of building interfaces to both support those who do not yet feel able to disclose, while promoting disclosure for the benefits it can provide. Yet, we find PLH participants who preferred to keep their status undisclosed developing 'signalling appropriation' strategies to help them take control over the disclosure of their status. For instance, identifying affordance properties in the privacy unraveling effect which allow them to subtly signal their status to others whilst simultaneously keeping it undisclosed; other PLH participants developed countersignals to reduce the effects of privacy unraveling around undisclosed HIV statuses. Finally, we draw from our findings together with previous work to propose a set of design considerations that could facilitate the use of signals to support the design of these sensitive disclosure fields.

\section{ACKNOWLEDGMENTS}

We would like to thank our study participants for their time and support of this research. This work is partially funded by the EU Horizon 2020 research and innovation program under the Marie Skłodowska-Curie Action ITN grant agreement No 675730 and the University College London (UCL) Department of Computer Science. 


\section{REFERENCES}

[1] 2018. Internet users, UK. Technical Report. Office for National Statistics (ONS). 1-8 pages. https://www.ons.gov.uk/businessindustryandtrade/ itandinternetindustry/bulletins/internetusers/2018/pdf

[2] George A Akerlof. 1978. The market for "lemons": Quality uncertainty and the market mechanism. In Uncertainty in Economics. Elsevier, 235-251.

[3] Nazanin Andalibi, Margaret Morris, and Andrea Forte. 2018. Testing Waters, Sending Clues: Indirect Disclosures of Socially Stigmatized Experiences on Social Media. Proceedings in ACM Human Computer Interaction CSCW (2018), 24. https://doi.org/10.1145/3274288

[4] Amid Ayobi, Paul Marshall, Anna L Cox, and Yunan Chen. 2017. Quantifying the body and caring for the mind: self-tracking in multiple sclerosis. In Proceedings of the 2017 CHI Conference on Human Factors in Computing Systems. ACM, 6889-6901.

[5] Hamdan Azhar. [n. d.]. How We Really Use The Peach. https: //blog.emojipedia.org/how-we-really-use-the-peach/

[6] Douglas G Baird, Robert H Gertner, and Randal C Picker. 1998. Game theory and the law. Harvard University Press.

[7] Leslie A Baxter. 1992. Root metaphors in accounts of developing romantic relationships. Fournal of Social and Personal Relationships 9 , 2 (1992), 253-275.

[8] Volker Benndorf, Dorothea Kübler, and Hans-Theo Normann. 2015. Privacy concerns, voluntary disclosure of information, and unraveling: An experiment. European Economic Review 75 (2015), 43-59.

[9] Daniela Bezemer, Frank de Wolf, Maarten C Boerlijst, Ard van Sighem, T Deirdre Hollingsworth, Maria Prins, Ronald B Geskus, Luuk Gras, Roel A Coutinho, and Christophe Fraser. 2008. A resurgent HIV-1 epidemic among men who have sex with men in the era of potent antiretroviral therapy. Aids 22, 9 (2008), 1071-1077.

[10] Walter O. Bockting, Michael H. Miner, Rebecca E. Swinburne Romine, Autumn Hamilton, and Eli Coleman. 2013. Stigma, mental health, and resilience in an online sample of the US transgender population. American fournal of Public Health 103, 5 (2013), 943-951. https://doi. org/10.2105/AJPH.2013.301241

[11] Kirsten Boehner and Jeffrey T Hancock. 2006. Advancing ambiguity. In Proceedings of the SIGCHI conference on Human Factors in computing systems. ACM, 103-106.

[12] Virginia Braun and Victoria Clarke. 2008. Using thematic analysis in psychology. Qualitative Research in Psychology 3, October 2012 (2008), 37-41. https://doi.org/10.1191/1478088706qp063oa

[13] AE Brown, S Nash, N Connor, PD Kirwan, D Ogaz, S Croxford, D De Angelis, and VC Delpech. 2017. Towards elimination of HIV transmission, AIDS and HIV-related deaths in the UK. HIV medicine (2017).

[14] Lisanne Brown, Kate Macintyre, and Lea Trujillo. 2003. Interventions To Reduce Hiv / Aids Stigma : What Have We Learned ? 15, 1 (2003), 49-69.

[15] Adrian Bussone, Simone Stumpf, and George Buchanan. 2016. It Feels Like I'm Managing Myself: HIV+ People Tracking Their Personal Health Information. In Proceedings of the 9th Nordic Conference on Human-Computer Interaction. ACM, 55.

[16] Sarah K Calabrese and Kristen Underhill. 2015. How stigma surrounding the use of HIV preexposure prophylaxis undermines prevention and pleasure: a call to destigmatize "truvada whores". American journal of public health 105, 10 (2015), 1960-1964.

[17] Alex Carballo-Diéguez, Michael Miner, Curtis Dolezal, B. R Simon Rosser, and Scott Jacoby. 2006. Sexual negotiation, HIV-status disclosure, and sexual risk behavior among latino men who use the internet to seek sex with other men. Archives of Sexual Behavior 35, 4 (2006), 473-481. https://doi.org/10.1007/s10508-006-9078-7
[18] ChingChe J. Chiu and Sean D. Young. 2015. The Relationship Between Online Social Network Use, Sexual Risk Behaviors, and HIV Sero-Status Among a Sample of Predominately African American and Latino Men Who have Sex with Men (MSM) Social Media Users. AIDS and Behavior 19, S2 (2015), 98-105. https://doi.org/10.1007/s10461-014-0986-6

[19] Judith A. Clair, Joy E. Beatty, and Tammy L. Maclean. 2005. Out of sight but not out of mind: Managing invisible social identities in the workplace. Academy of Management Review 30, 1 (2005), 78-95. https://doi.org/10.5465/AMR.2005.15281431

[20] Jennifer L Doleac and Benjamin Hansen. 2016. Does "ban the box" help or hurt low-skilled workers? Statistical discrimination and employment outcomes when criminal histories are hidden. Technical Report. National Bureau of Economic Research.

[21] Judith Donath. 2006. Signals, cues and meaning. Unpublished manuscript. (2006), 1-27. https://doi.org/10.1017/CBO9781107415324.004 arXiv:arXiv:1011.1669v3

[22] Judith S Donath. "In press". Signals, Truth, and Design. MIT Press., Cambridge, MA.

[23] Timothy Edgar. 1994. Self-Disclosure Behaviors of the Stigmatized: Strategies and Outcomes for the Revelation of Sexual Orientation. In Queer words, queer images: Communication and the construction of homosexuality (Jeffery Ringer). 221-237 pages.

[24] Nicole Ellison, Rebecca Heino, and J. L. Gibbs. 2006. Managing Impressions Online: Self-Presentation Processes in the Online Dating Environment. fournal of Computer-Mediated Communication 11 (2006), 415-441. https://doi.org/10.1111/j.1083-6101.2006.00020.x

[25] Nicole B Ellison and Jeffrey T Hancock. 2013. Profile as promise: Honest and deceptive signals in online dating. IEEE Security \& Privacy 5 (2013), 84-88.

[26] Nick Feltovich, Richmond Harbaugh, and Ted To. 2002. Too Cool for School? Signalling and Countersignalling. The RAND fournal of Economics 33, 4 (2002), 630. https://doi.org/10.2307/3087478

[27] Sheila L Fesko. 2001. Disclosure of HIV Status in The Workplace. Health \& Social Work 26, 4 (2001), 235.

[28] Andrew T Fiore, Lindsay Shaw Taylor, Gerald A Mendelsohn, and Marti Hearst. 2008. Assessing attractiveness in online dating profiles. Proceeding of the twenty-sixth annual CHI conference on Human factors in computing systems - CHI '08 1992 (2008), 797. https://doi.org/10. $1145 / 1357054.1357181$

[29] Robert H Frank and Ian C Parker. 1991. Microeconomics and behavior. McGraw-Hill New York.

[30] William W Gaver, Jacob Beaver, and Steve Benford. 2003. Ambiguity as a resource for design. In Proceedings of the SIGCHI conference on Human factors in computing systems. ACM, 233-240.

[31] Jennifer L. Gibbs, Nicole B. Ellison, and Rebecca D. Heino. 2006. SelfPresentation in Online Personals. Communication Research 33, 2 (2006), 152-177. https://doi.org/10.1177/0093650205285368

[32] Erving Goffman. 1956. The presentation of self in everyday life. Vol. 21. xii, 259 p. pages. https://doi.org/10.2307/258197

[33] Erving Goffman. 1963. Stigma. Notes on the management of spoiled identity. , 147 pages. https://doi.org/10.2307/2091442 arXiv:arXiv:1011.1669v3

[34] Sarit A. Golub. 2018. PrEP Stigma: Implicit and Explicit Drivers of Disparity. Current HIV/AIDS Reports 15, 2 (2018), 190-197. https: //doi.org/10.1007/s11904-018-0385-0

[35] Oliver L Haimson, Anne E Bowser, Edward F Melcer, and Elizabeth F Churchill. 2015. Online Inspiration and Exploration for Identity Reinvention. CHI '15 - Proceedings of the ACM Conference on Human Factors in Computing Systems 1 (2015), 3809-3818. https: //doi.org/10.1145/2702123.2702270

[36] H Irene Hall, David R Holtgrave, and Catherine Maulsby. 2012. HIV transmission rates from persons living with HIV who are aware and 
unaware of their infection. Aids 26, 7 (2012), 893-896.

[37] Mark J. Handel and Irina Shklovski. 2012. Disclosure, ambiguity and risk reduction in real-time dating sites. Proceedings of the 17th ACM international conference on Supporting group work - GROUP '12 (2012), 175. https://doi.org/10.1145/2389176.2389203

[38] Roma Harris, Tiffany Veinot, Leslie Bella, Irving Rootman, and Judith Krajnak. 2008. Helpers, gatekeepers and the well-intentioned: The mixed blessings of HIV/AIDS info (r) mediation in rural Canada. In Mediating Health Information. Springer, 167-181.

[39] Rebecca D Heino, Nicole B Ellison, and Jennifer L Gibbs. 2010. Relationshopping: Investigating the market metaphor in online dating. fournal of Social and Personal relationships 27, 4 (2010), 427-447.

[40] Gregory M Herek. 2014. HIV-related stigma. (2014).

[41] B. Hogan. 2010. The Presentation of Self in the Age of Social Media: Distinguishing Performances and Exhibitions Online. Bulletin of Science, Technology \& Society 30, 6 (2010), 377-386. https: //doi.org/10.1177/0270467610385893 arXiv:arXiv:1011.1669v3

[42] Seoyeon Hong, Edson Tandoc, Eunjin Anna Kim, Bokyung Kim, and Kevin Wise. 2012. The Real You? The Role of Visual Cues and Comment Congruence in Perceptions of Social Attractiveness from Facebook Profiles. Cyberpsychology, Behavior, and Social Networking 15, 7 (2012), 339-344. https://doi.org/10.1089/cyber.2011.0511

[43] Rusi Jaspal and Christos Daramilas. 2016. Perceptions of pre-exposure prophylaxis (PrEP) among HIV-negative and HIV-positive men who have sex with men (MSM). Cogent Medicine 3, 1 (2016), 1-16. https: //doi.org/10.1080/2331205X.2016.1256850

[44] Rusi Jaspal and Iain Williamson. 2017. Identity management strategies among HIV-positive Colombian gay men in London. Culture, Health \& Sexuality 1058, June (2017), 1-15. https://doi.org/10.1080/13691058. 2017.1314012

[45] Anirudha Joshi, Mandar Rane, Debjani Roy, Nagraj Emmadi, Padma Srinivasan, N Kumarasamy, Sanjay Pujari, Davidson Solomon, Rashmi Rodrigues, DG Saple, et al. 2014. Supporting treatment of people living with HIV/AIDS in resource limited settings with IVRs. In Proceedings of the SIGCHI Conference on Human Factors in Computing Systems. ACM, 1595-1604.

[46] S J Klein, W D Karchner, and D A O'Connell. 2002. Interventions to prevent HIV-related stigma and discrimination: findings and. F Public Health Manag Pract 8, 6 (2002), 44-53.

[47] Cliff Lampe, Nicole Ellison, and Charles Steinfield. 2007. A Familiar Face(book): Profile Elements as Signals in an Online Social Network. Proceedings of the SIGCHI conference on Human factors in computing systems - CHI 2007 (2007), 435-444. https://doi.org/10.1145/1240624. 1240695

[48] Soohyung Lee and Muriel Niederle. 2014. Propose with a rose? Signaling in internet dating markets. Experimental Economics 18, 4 (2014), 731-755. https://doi.org/10.1007/s10683-014-9425-9

[49] Karen Levy and Solon Barocas. 2017. Designing against discrimination in online markets. Berkeley Technology Law fournal 32 (2017). https: //ssrn.com/abstract $=3084502$

[50] Gregory Lewis. 2011. Asymmetric information, adverse selection and online disclosure: The case of eBay motors. American Economic Review 101, 4 (2011), 1535-46.

[51] James D Livingston, Teresa Milne, Mei Lan Fang, and Erica Amari. 2012. The effectiveness of interventions for reducing stigma related to substance use disorders: a systematic review. Addiction 107, 1 (2012), 39-50.

[52] Juan F Maestre, Elizabeth V Eikey, Mark Warner, Svetlana Yarosh, Jessica Pater, Maia Jacobs, Gabriela Marcu, and Patrick C Shih. 2018. Conducting Research with Stigmatized Populations: Practices, Challenges, and Lessons Learned. In Companion of the 2018 ACM Conference on Computer Supported Cooperative Work and Social Computing. ACM,
385-392

[53] JAMES Mallet. 1993. Speciation, raciation, and color pattern evolution in Heliconius butterflies: evidence from hybrid zones. Hybrid zones and the evolutionary process (1993), 226-260.

[54] Gabriela Marcu, Nadia Dowshen, Shuvadittya Saha, Ressa Reneth Sarreal, and Nazanin Andalibi. 2016. TreatYoSelf: Empathy-driven behavioral intervention for marginalized youth living with HIV. Proceedings of the International Conference on Pervasive Computing Technologies for Healthcare (PervasiveHealth 2016) (2016), 1-8. https: //doi.org/10.4108/eai.16-5-2016.2263336

[55] Alice E Marwick and Danah Boyd. 2011. I tweet honestly, I tweet passionately: Twitter users, context collapse, and the imagined audience. New media \& society 13, 1 (2011), 114-133.

[56] Christina Masden and W Keith Edwards. 2015. Understanding the Role of Community in Online Dating. Proceedings of the 33rd Annual ACM Conference on Human Factors in Computing Systems - CHI '15 (2015), 535-544. https://doi.org/10.1145/2702123.2702417

[57] Sarah McRoberts, Haiwei Ma, Andrew Hall, and Svetlana Yarosh. 2017. Share First, Save Later: Performance of Self through Snapchat Stories. In Proceedings of the 2017 CHI Conference on Human Factors in Computing Systems - CHI '17. 6902-6911. https://doi.org/10.1145/3025453. 3025771

[58] Rebecca J Meisenbach. 2010. Stigma management communication: A theory and agenda for applied research on how individuals manage moments of stigmatized identity. Fournal of Applied Communication Research 38, 3 (2010), 268-292. https://doi.org/10.1080/00909882.2010. 490841

[59] Laura Nyblade, Anne Stangl, Ellen Weiss, and Kim Ashburn. 2009. Combating HIV stigma in health care settings: What works? Journal of the International AIDS Society 12, 1 (2009). https://doi.org/10.1186/ 1758-2652-12-15

[60] Alex Pentland. 2008. Understanding 'Honest Signals' in business. October 50, 1 (2008), 70-75.

[61] Scott R Peppet. 2011. Unraveling privacy: The personal prospectus and the threat of a full-disclosure future. Nw. UL Rev. 105 (2011), 1153.

[62] Jennifer L Peterson. 2010. The challenges of seeking and receiving support for women living with HIV. Health Communication 25, 5 (2010), 470-479.

[63] Andrew N Phillips, Valentina Cambiano, Fumiyo Nakagawa, Alison E Brown, Fiona Lampe, Alison Rodger, Alec Miners, Jonathan Elford, Graham Hart, Anne M Johnson, et al. 2013. Increased HIV incidence in men who have sex with men despite high levels of ART-induced viral suppression: analysis of an extensively documented epidemic. PloS one 8, 2 (2013), e55312.

[64] Steven D. Pinkerton and Carol L. Galletly. 2007. Reducing HIV transmission risk by increasing serostatus disclosure: A mathematical modeling analysis. AIDS and Behavior 11, 5 (2007), 698-705. https://doi.org/10.1007/s10461-006-9187-2 arXiv:NIHMS150003

[65] Nithya Ramanathan, Dallas Swendeman, W Scott Comulada, Deborah Estrin, and Mary Jane Rotheram-Borus. 2013. Identifying preferences for mobile health applications for self-monitoring and selfmanagement: Focus group findings from HIV-positive persons and young mothers. International journal of medical informatics 82, 4 (2013), e38-e46.

[66] Nithya Ramanathan, Dallas Swendeman, W Scott Comulada, Deborah Estrin, and Mary Jane Rotheram-Borus. 2013. Identifying preferences for mobile health applications for self-monitoring and selfmanagement: Focus group findings from HIV-positive persons and young mothers. International journal of medical informatics 82, 4 (2013), e38-e46.

[67] Alison Rodger, Tina Bruun, Valentina Cambiano, Pietro Vernazza, V Strada, Jan Van Lunzen, et al. 2014. 153LB: HIV transmission risk 
through condomless sex if HIV+ partner on suppressive ART: PARTNER Study. In 21st Conference on Retroviruses and Oppotunistic Infections. 3-6.

[68] Alison Rodger, Valentina Cambiano, Tina Bruun, Pietro Vernazza, Simon Collins, Olaf Degen, et al. 2018. HIV transmission risk through condomless sex in gay couples with suppressive ART: The PARTNER2 Study extended results in gay men. (2018). https://www.chip.dk/ Studies/PARTNER/PARTNER-press-release-2018 AIDS2018.

[69] Gabrielle M Salib, Juan Fernando Maestre, Kenneth B Nimley, Nadia Dowshen, and Gabriela Marcu. 2018. The Role of Reflection and Context in Medication Adherence Tracking for People Living with HIV. Extended Abstracts of the 2018 CHI Conference on Human Factors in Computing Systems (2018), LBW042:1--LBW042:6. https://doi.org/ $10.1145 / 3170427.3188631$

[70] Julianne Serovich, Sandra Reed, Judy Kimberly, and Dana Putney. 2014 Disclosure strategies, comfort with disclosure, and HIV transmission risk among HIV-positive MSM. Sexually Transmitted Diseases 41 (2014), 82639.

[71] Julianne M Serovich, Daniel G Oliver, Sarah a Smith, and Tina L Mason. 2005. Methods of HIV disclosure by men who have sex with men to casual sexual partners. AIDS patient care and STDs 19, 12 (2005), 823832. https://doi.org/10.1089/apc.2005.19.823

[72] N. Sadat Shami, Kate Ehrlich, Geri Gay, and Jeffrey T. Hancock. 2009. Making sense of strangers' expertise from signals in digital artifacts. Proceedings of the SIGCHI conference on Human factors in computing systems - CHI'09 (2009), 69. https://doi.org/10.1145/1518701.1518713

[73] R Smith, K Rossetto, and B L Peterson. 2008. A meta-analysis of disclosure of one's HIV-positive status, stigma and social support. AIDS Care 20, 10 (2008), 1266-1275.

[74] A Michael Spence. 1973. Time and communication in economic and social interaction. The Quarterly fournal of Economics 87, 4 (1973), 651-660.

[75] stigmaindexuk.org. 2015. The People Living with HIV Stigma Survey UK. Men Who Have Sex With Men (MSM).

[76] Catalina L Toma and Jeffrey T Hancock. 2010. Reading between the Lines : Linguistic Cues to Deception in Online Dating Profiles. Proceedings of the 2010 ACM conference on Computer supported cooperative work - CSCW'10 (2010). https://doi.org/10.1145/1718918.1718921

[77] Suvi Uski and Airi Lampinen. 2016. Social norms and self-presentation on social network sites: Profile work in action. New Media \& Society 18, 3 (2016), 447-464. https://doi.org/10.1177/1461444814543164

[78] Tiffany Veinot. 2009. "A lot of people didn't have a chance to support us because we never told them" Stigma management, information poverty and HIV/AIDS information/help networks. Proceedings of the American Society for Information Science and Technology 46, 1 (2009), $1-20$.

[79] Tiffany C Veinot. 2010. We have a lot of information to share with each other": Understanding the value of peer-based health information exchange. Information Research 15, 4 (2010), 15-4.

[80] Tiffany C. Veinot and Roma Harris. 2011. Talking About, Knowing About HIV/AIDS in Canada: A Rural-Urban Comparison. fournal of Rural Health 27, 3 (2011), 310-318. https://doi.org/10.1111/j.1748-0361. 2010.00353.x

[81] Joseph B Walther, Brandon Van Der Heide, Lauren M Hamel, and Hillary C Shulman. 2009. Self-generated versus other-generated statements and impressions in computer-mediated communication: A test of warranting theory using Facebook. Communication research 36, 2 (2009), 229-253.

[82] M Warner, A Gutmann, MA Sasse, and A Blandford. 2018. Privacy Unraveling Around Explicit HIV Status Disclosure Fields in the Online Geosocial Hookup App Grindr. Proceedings of the ACM on HumanComputer Interaction 2 (November 2018). Issue CSCW. https://doi.org/
$10.1145 / 3274450$

[83] Stacey L Williams and Kristin D Mickelson. 2008. A paradox of support seeking and rejection among the stigmatized. Personal Relationships 15, 4 (2008), 493-509.

[84] Sarah Wiseman and Sandy J J Gould. 2018. Repurposing Emoji for Personalised Communication: Why ? means "I love you". Proceedings of the SIGCHI conference on Human factors in computing systems - CHI'18 (2018), 1-10. https://doi.org/10.1145/3173574.3173726

[85] Dan Wohlfeiler, Jennifer Hecht, Jonathan Volk, H. Fisher Raymond, Tom Kennedy, and Willi McFarland. 2013. How can we improve online HIV and STD prevention for men who have sex with men? perspectives of hook-up website owners, website users, and HIV/STD directors. AIDS and Behavior 17, 9 (2013), 3024-3033. https://doi.org/10.1007/ s10461-012-0375-y arXiv:NIHMS150003

[86] Jennifer Yurchisin, Kittichai Watchravesringkan, and Deborah Brown Mccabe. 2005. An Exploration of Identity Re-Creation in the Context of Internet Dating. Social Behavior and Personality: an international journal 33, 8 (2005), 735-750. https://doi.org/10.2224/sbp.2005.33.8.735

[87] Amotz Zahavi. 1975. Mate selection-A selection for a handicap. fournal of Theoretical Biology 53, 1 (1975), 205-214. https://doi.org/10.1016/ 0022-5193(75)90111-3

[88] Shanyang Zhao, Sherri Grasmuck, and Jason Martin. 2008. Identity construction on Facebook: Digital empowerment in anchored relationships. Computers in Human Behavior 24, 5 (2008), 1816-1836. https://doi.org/10.1016/j.chb.2008.02.012

[89] Douglas Zytko, Sukeshini A Grandhi, and Quentin Gad Jones. 2014. Impression management through communication in online dating. Proceedings of the companion publication of the 17th ACM conference on Computer supported cooperative work \& social computing. (2014), 277-280. https://doi.org/10.1145/2556420.2556487 